\title{
COVID-19 projections for reopening Connecticut
}

\author{
Forrest W. Crawford ${ }^{1,2,3,4}$, Zehang Richard $\mathrm{Li}^{1}$, and Olga Morozova ${ }^{1}$ \\ 1. Department of Biostatistics, Yale School of Public Health \\ 2. Department of Statistics \& Data Science, Yale University \\ 3. Department of Ecology \& Evolutionary Biology, Yale University \\ 4. Yale School of Management
}

May 20, 2020

\section{Key Points:}

- Closure of schools and the statewide "Stay Safe, Stay Home" order have effectively reduced COVID-19 transmission in Connecticut, with model projections estimating incidence at about 1,300 new infections per day.

- If close interpersonal contact increases quickly in Connecticut following reopening on May 20, the state is at risk of a substantial increase of COVID-19 infections, hospitalizations, and deaths by late Summer 2020.

- Real-time metrics including case counts, hospitalizations, and deaths may fail to provide enough advance warning to avoid resurgence.

- Substantial uncertainty remains in our knowledge of cumulative COVID-19 incidence, the proportion of infected individuals who are asymptomatic, infectiousness of children, the effects of testing and contact tracing on isolation of infected individuals, and how contact patterns may change following reopening.

\section{Introduction}

Connecticut is one of the US states most severely impacted by the COVID-19 pandemic, with over 37,000 cases, 11,000 hospitalizations, and 3,400 deaths [1,2]. On March 8, 2020, the first Connecticut COVID-19 case was identified, and the state began interventions to slow transmission in mid March 2020. On March 17, Governor Ned Lamont ordered all in-person classes at K-12 schools canceled [3], and later extended the closure for the remainder of the 2019-2020 academic year [4-6]. The Governor issued a statewide "Stay Safe, Stay Home" order to take effect on March 23 [7]. Evidence from device mobility data suggests that Connecticut residents reduced travel outside the home, and increased the number of hours per day spent at home [8,9]. At the same time, the Governor ordered businesses closed, with the exception of essential businesses that could remain open with additional restrictions and guidelines to minimize close contact and risk of transmission.

The number of hospitalized COVID-19 patients in Connecticut peaked in mid April, and began a slow decline. In early May, Governor Lamont issued plans and guidance for reopening the state, a process that began on May 20. The Connecticut Department of Economic and Community Development issued rules and guidelines for opening particular business sectors [10]. Monitoring COVID-19 test results, expanding access to testing, and contact tracing are part of the state's intervention strategy. Contact tracing will be implemented using the state's ContaCT platform 
medRxiv preprint doi: https://doi.org/10.1101/2020.06.16.20126425; this version posted June 19, 2020. The copyright holder for this preprint (which was not certified by peer review) is the author/funder, who has granted medRxiv a license to display the preprint in perpetuity.

It is made available under a CC-BY 4.0 International license .

[11], and the state publishes daily testing reports [e.g. 12]. The Governor announced goals to scale up COVID-19 viral testing to 42,000 tests per day [13, 14], but testing capacity is still limited [15].

As Connecticut reopens, policymakers need access to reliable information about both the current state of the pandemic and likely future outcomes. Elected officials and public health agencies already have access to near real-time information about COVID-19 testing, case counts, hospitalizations, and deaths. However, these data may not provide timely insight into the current and future dynamics of COVID-19 transmission. Because viral testing of asymptomatic individuals is not yet widespread, symptomatic individuals at least 4-5 days from infection may constitute the majority who seek testing [16-22], and COVID-19 case counts or positive test proportion may be poor surrogate measures of current infection prevalence. Hospitalization may not occur until more than 2 or 3 weeks following infection, and death may not occur for weeks after that [17, 23-27]. Furthermore, current data may not provide information about what is likely to occur under the Governor's stated reopening plans or interventions to be implemented in the future. As the state scales up its testing capacity, expands contact tracing, and initiates phased reopening of businesses, historical data on cases, hospitalizations, and deaths may not give insight into the possible effects of these interventions on future incidence. Finally, early warning systems based on these lagging metrics may fail to provide state policymakers with the predictive information they need to initiate a reversion to a more restrictive phase or implement other interventions to blunt a coming wave of new infections.

In this report, we use a mathematical infectious disease transmission model to project COVID-19 incidence, hospitalizations, and deaths in the state of Connecticut through August 31, 2020. We consider population-level contact scenarios informed by the Connecticut Governor's phased reopening plans. Model parameters are calibrated using data on hospitalizations and deaths in Connecticut and estimates from the literature on clinical epidemiology of COVID-19. A separate technical report describes the data, transmission model, and calibration in detail [28]. The primary purpose of this report is to provide state and local decision-makers with information they can use to plan reopening of the state in a way that minimizes the risk of a resurgence in COVID-19 cases, hospitalizations, and deaths. The secondary purpose of this report is to assist state agencies and non-governmental organizations in implementation of public health responses, including testing, contact tracing, and design of incidence, infection prevalence, and seroprevalence studies.

\section{COVID-19 projections: March 1 to August 31, 2020}

We present projections of COVID-19 incidence, hospitalizations, and deaths from March 1 to August 31, 2020. Projections and $95 \%$ uncertainty intervals show the range of possible outcomes over the Summer under "slow" and "fast" reopening scenarios. Projections prior to May 20, 2020 are calibrated to historical hospitalization and death data obtained from the Connecticut Department of Public Health [29]. County-level hospitalization and bed capacity are obtained from the Connecticut Hospital Association and CHIMEData [30, 31]. This analysis does not use individual-level patient data. Projections beyond May 20 depend on assumptions about the nature of contact and viral testing in the future, under the Governor's plan for reopening the state [32].

A separate technical report describes the data, transmission model, calibration, and uncertainty calculation in greater detail [28]. Briefly, we employ a modified compartmental susceptible-exposed-infectious-removed (SEIR) transmission model that accommodates asymptomatic, mildly symptomatic, and severe cases, with additional compartments for hospitalizations, unhospitalized severe infections in the case of hospital overflow, patients in nursing homes and assisted living facilities, and deaths. Exposed individuals are assumed to become infectious 4 days after infection [16-22]. Mildly symptomatic individuals are assumed to self-isolate on average 4 days after they become infectious (or 2.5 days after they develop symptoms) [21, 33, 34], and subsequently remain in isolation until recovery. Asymptomatic individuals are assumed to be less transmissible compared to symptomatic cases, but remain infectious for an average of 7 days in the absence of widespread testing [35]. All severe cases are assumed to require hospitalization. Severe cases occurring in closed facilities, such as nursing homes or assisted living facilities, are tracked separately. 

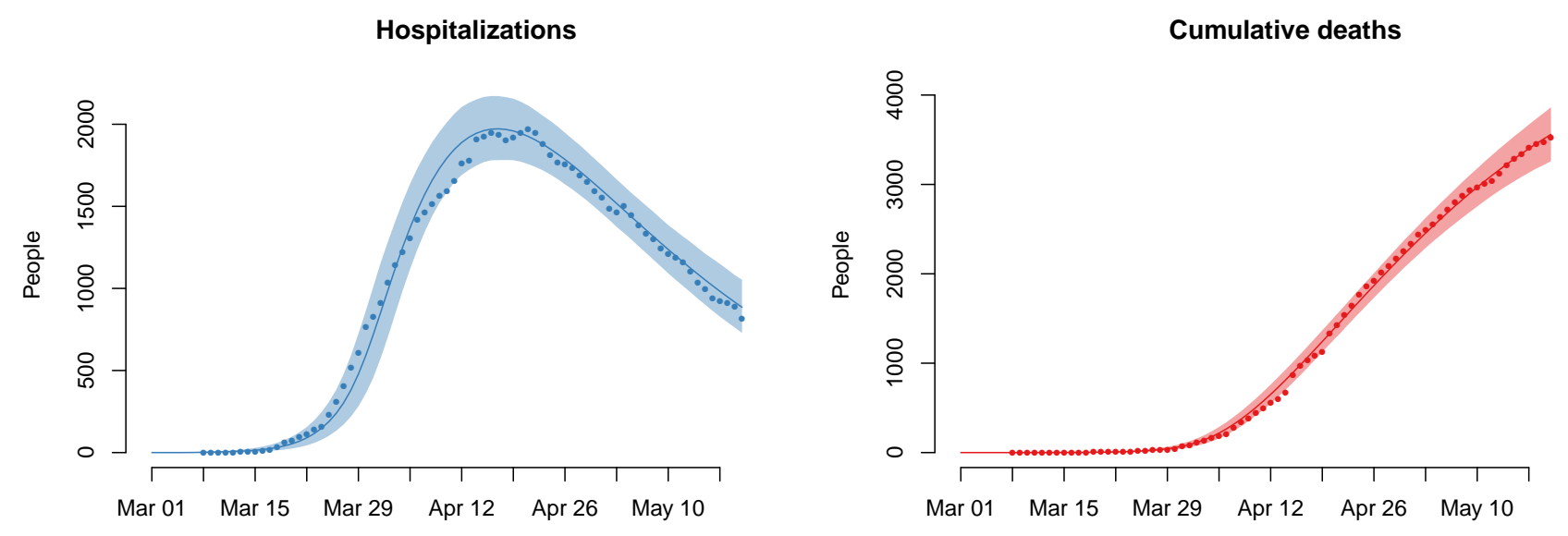

Figure 1: Model projections of current hospitalizations and cumulative deaths, with observed data, from March 1 to May 20, 2020. Solid lines show average model projections and the intervals show 95\% uncertainty regions. Observed data are overlaid as solid dots. The figure is reproduced from [28], doi: https://doi.org/10.1101/2020.06.12.20126391.

Only severe cases can result in death. We assume that COVID-19 infection confers lasting immunity to reinfection at least within the projection time interval of March 1 to August 31, 2020.

In this report we present results assuming 50\% of infections are asymptomatic, though there is still uncertainty about this quantity [20,36-40]. We assume that $10 \%$ of symptomatic infections are severe and require hospitalization $[17,21,23,41]$. Severe cases transition through a latent period, pre-hospitalization infectious period, hospitalization period, and eventually either recover or die. We assume that hospitalizations and deaths are reported with a lag and account for this lag when we match projections to observations. Reporting lags along with average time spent at various stages preceding hospitalization and death are calibrated to observations. Average reporting lag for hospitalizations is shorter than that of deaths due to the latter being a combination of deaths occurring in hospitals and in nursing facilities. Figure 1 shows current COVID-19 hospitalization census and cumulative death projections from March 1 to May 20, 2020. Projections match observed data well, and data points largely fall within prediction uncertainty regions.

Additional calibrated projections under low (36\%) and high (70\%) asymptomatic fractions are provided in a separate technical report [28]. Briefly, when asymptomatic fraction is high, we see more infections, hospitalizations and deaths by the end of Summer. Under slow reopening, hospitalization capacity is not expected to be overwhelmed under any of the scenarios. In case of fast reopening, hospitalization capacity would likely be exceeded sooner if the asymptomatic fraction is high. Differing assumptions about the asymptomatic fraction can result in large differences in estimated cumulative incidence proportion. By the end of Summer, cumulative incidence may be as low as 0.1 under low asymptomatic fraction with slow reopening, or as high as 0.6 under high asymptomatic fraction with fast reopening. The latter scenario predicts the second peak of infections to occur in mid-August, while all other scenarios predict that the second peak would not occur until later in the Fall. While a higher asymptomatic fraction would result in a higher number of deaths in the short-term, the total number of deaths by the end of the epidemic would likely be higher if the asymptomatic fraction is low.

Though we present projections for the state of Connecticut as a whole, the COVID-19 transmission model is structured by county, with transmission allowed between adjacent counties. Reopening scenarios are represented in the projection model by a schedule of proportional increases in contact, beginning on the stated reopening date of May 20, 2020. Slow versus fast reopening scenarios are parameterized by monthly versus biweekly release of $10 \%$ of suppressed contact. We estimate that the combined effect of school closure and the "Stay Safe, Stay Home" order reduced contact by about $85 \%$ below baseline. Following reopening, viral testing and contact tracing are assumed to increase the rate of isolation of infected individuals. In particular, we assume that asymptomatic individuals are 


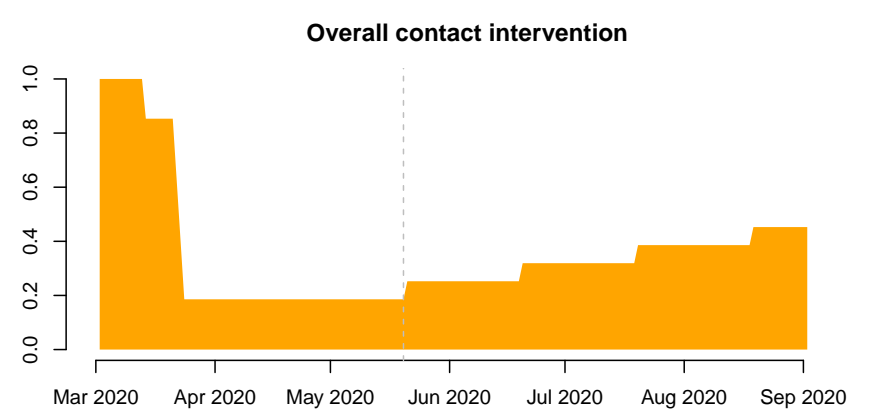

Required hospitalizations in Connecticut

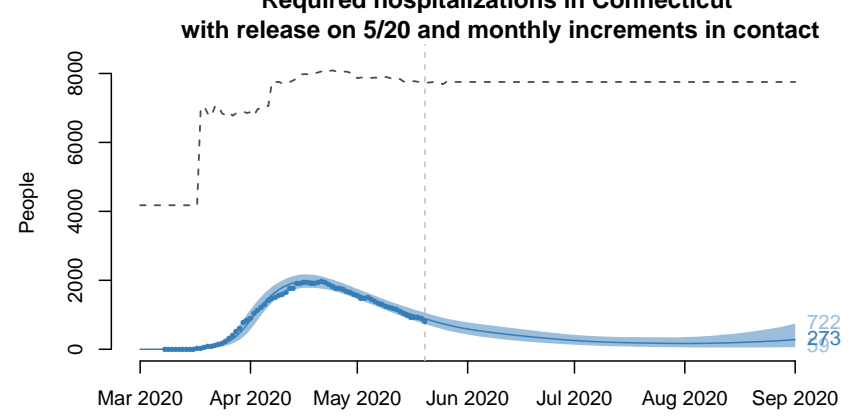

Daily new infections in Connecticut
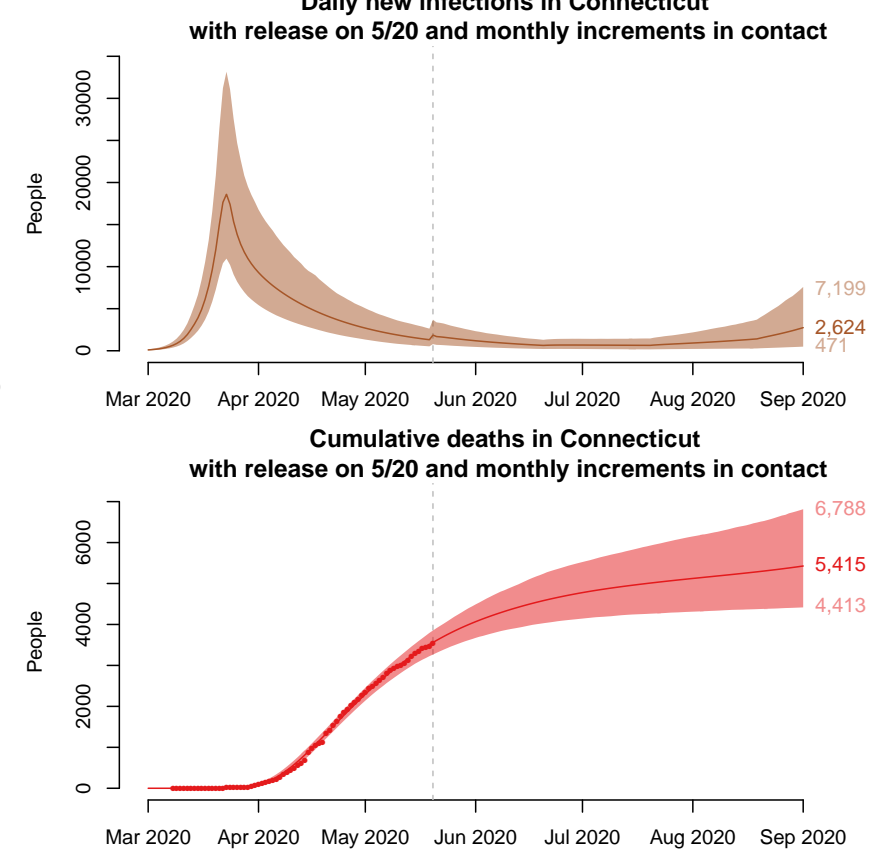

Figure 2: Projections under a "slow" reopening scenario in which $10 \%$ of suppressed contact is released every 30 days, starting on May 20, 2020, with 95\% uncertainty intervals. Clockwise from top left: population-level historical and projected contact patterns, daily COVID-19 incidence, hospital census, and deaths. The dashed line above hospitalization projections is an estimate of the hospital bed capacity in Connecticut [30]. The figure is reproduced from [28], doi: https://doi.org/10.1101/2020.06.12.20126391.

infectious for an average of 7 days, and mildly symptomatic cases are infectious for 4 days, of which 1.5 days is assumed to constitute a pre-symptomatic infectious period [21, 22, 33-35]. Expanded testing and contact tracing, implemented in the weeks following reopening, are assumed to reduce the average duration of infectiousness of asymptomatic individuals to 5.8 days and mildly symptomatic to 2.7 days.

\section{Projections under slow reopening}

Figure 2 shows projections of daily incidence, hospitalizations census, and cumulative deaths under a "slow" reopening scenario. At one-month intervals, $10 \%$ of the suppressed contact (relative to March 1 baseline) is released. In these projections, contact rates are at baseline in early March, dropping an estimated 85\% of contacts due to closure of schools and the "Stay Safe, Stay Home" order. Under this slow reopening scenario, daily incidence increases following reopening, but decays and stays low until mid August. Hospital census continues its gradual decline through June, flattening in July, and rising again slightly in August. Deaths rise slowly, with the greatest increases occurring in August. Between 4,400 and 6,800 deaths are projected by September 1.

\section{Projections under fast reopening}

Figure 3 shows projections of daily incidence, hospitalizations census, and cumulative deaths under a "fast" reopening scenario. At two-week intervals, $10 \%$ of the suppressed contact is released. In contrast to the "slow" scenario described above, here contact increases rapidly, and by the end of July, daily incidence rises above levels seen in March. By the beginning of September, hospital capacity may be exceeded, resulting in a sharp rise in deaths. Though there is uncertainty about the speed and timing of this predicted rise in hospitalizations and deaths in July-August 


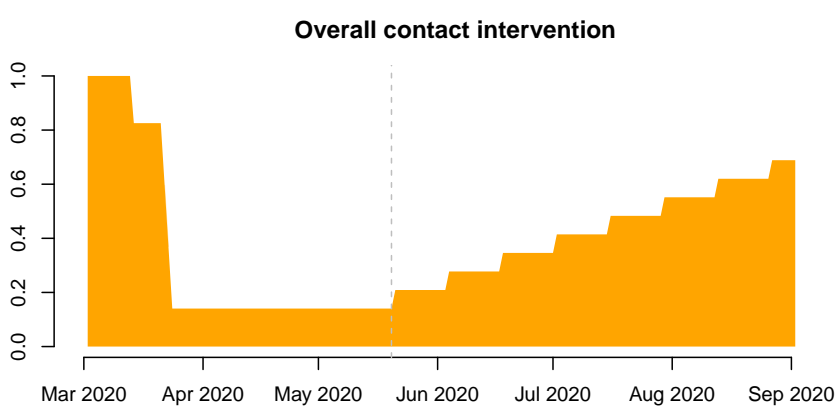

Required hospitalizations in Connecticut with release on $5 / 20$ and biweekly increments in contact

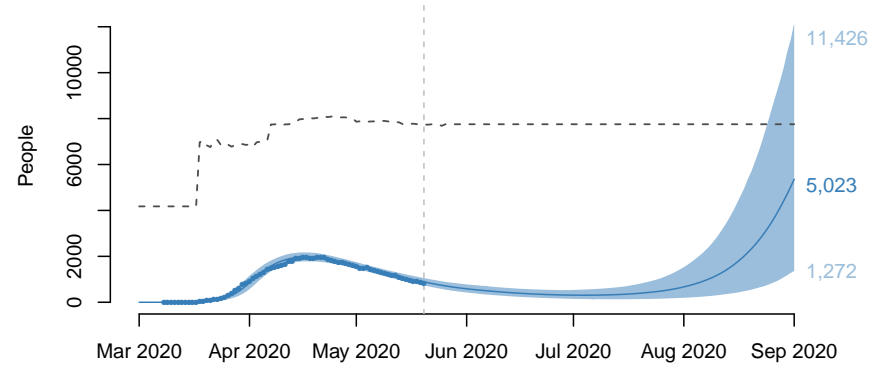

Daily new infections in Connecticut with release on $\mathbf{5 / 2 0}$ and biweekly increments in contact

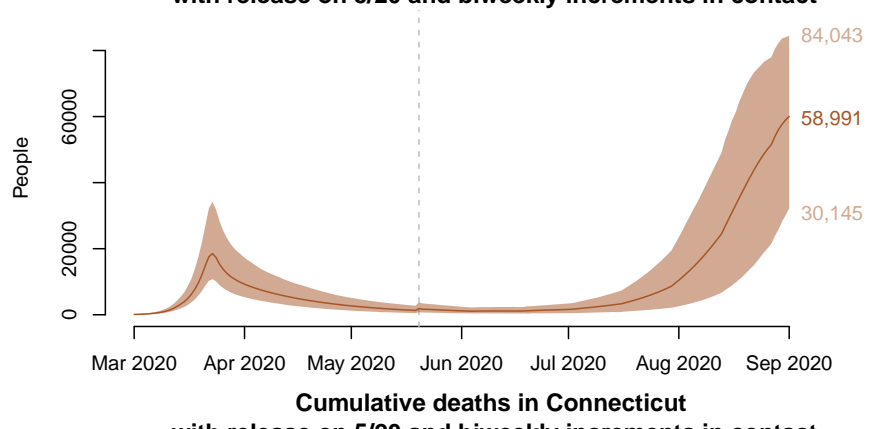
with release on $5 / 20$ and biweekly increments in contact

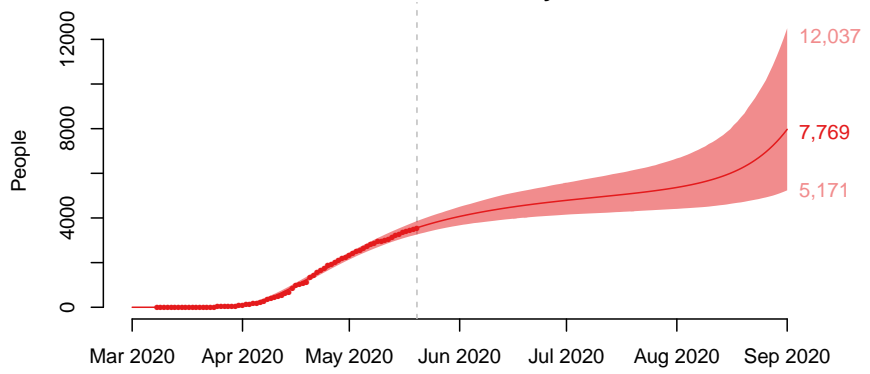

Figure 3: Projections under a "fast" reopening scenario in which $10 \%$ of suppressed contact is released every 14 days, starting on May 20, 2020, with 95\% uncertainty intervals. Clockwise from top left: population-level historical and projected contact patterns, daily COVID-19 incidence, hospital census, and deaths. The dashed line above hospitalization projections is an estimate of the hospital bed capacity in Connecticut [30], which may be exceeded during August in this scenario. The figure is reproduced from [28], doi: https://doi.org/10.1101/2020.06. 12.20126391 .

under a fast reopening scenario, the second wave is likely to occur. Between 5,200 and 12,000 deaths are projected by September 1 .

\section{Implications}

The COVID-19 outbreak in Connecticut is currently declining, with hospitalization census decreasing on most days since mid April. Daily incidence at the end of May is estimated to be 1,309 (95\% uncertainty interval 505-2,720) new infections per day. However, as the state reopens, there is a risk that increased contact will result in a second wave of COVID-19 transmission, leading to an increase in cases, hospitalizations and deaths. If contact remains low throughout the Summer, with only minimal increases due to reopening, a possible second wave will be blunted and pushed into Fall of 2020. On the other hand, if contact returns quickly to a level seen in early March, then a sharp resurgence in hospitalizations and deaths is likely to occur in August. We do not know with certainty how contact patterns in the Connecticut population will change following reopening on May 20, but it is likely that the true pattern will fall between the slow and fast reopening scenarios described in this report.

\section{Risk of resurgence following reopening}

Projections under a fast reopening scenario suggest a rapid rise in new infections in June and July, followed by an increase in hospitalizations and deaths in August. If transmission increases quickly following reopening, indications of this increase might not be apparent in case counts and hospital data until mid to late July, as the first 
medRxiv preprint doi: https://doi.org/10.1101/2020.06.16.20126425; this version posted June 19, 2020. The copyright holder for this preprint (which was not certified by peer review) is the author/funder, who has granted medRxiv a license to display the preprint in perpetuity.

It is made available under a CC-BY 4.0 International license .

post-reopen infections become hospitalizations. Therefore it is important for public health authorities to closely monitor new hospitalizations as well as hospital census for signs of increased numbers of COVID-19 cases requiring healthcare. Transmission model projections like the ones presented here may provide more timely warnings than contemporaneous data on cases.

\section{Importance of testing to detect changes in incidence and isolate infectious individuals}

Enhanced viral testing, especially of asymptomatic individuals, may help provide an early warning of increased transmission following reopening. Because there is a roughly 3-week lag between a new infection and possible hospitalization of the infected individual, widespread and frequent testing could detect increased incidence before the first new symptomatic cases present to the hospital. Daily new infections are not observable in real time, but can be estimated using model projections like those presented here. In addition to its usefulness as an early warning system, widespread testing of symptomatic and asymptomatic people, followed by contact tracing, also constitutes a public health intervention. When positive individuals isolate themselves following receipt of a positive test result, they shorten the time during which they expose their contacts to their infection. In this way, testing and contact tracing can hasten isolation of infectious individuals, thereby reducing population-level transmission. In the scenarios presented above, we assume that testing and contact tracing shorten the average infectious period of all asymptomatic individuals from 7 to 5.8 days, and the infectious period of mildly symptomatic individuals from 4 days to 2.7 days. However, widespread testing, contact tracing, and subsequent isolation of individuals who test positive could further reduce the time interval of possible transmission and thereby further suppress incidence.

\section{Model projections to inform seroprevalence studies}

Expanded and accurate antibody testing in Connecticut could dramatically improve knowledge about seroprevalence - the proportion of people in Connecticut who have evidence of prior COVID-19 infection. If this proportion were more precisely known, the fraction of infected individuals who are asymptomatic could be estimated with greater certainty. Currently available data on deaths, hospitalizations and registered cases is consistent with a wide range of asymptomatic infections proportions. This knowledge would reduce uncertainty in future projections because it would reduce uncertainty about the number of people who have already been infected. Approximating cumulative incidence by the cumulative number of registered cases may be seriously misleading, because it omits individuals who experienced asymptomatic infection, or those who were never tested. If infection confers lasting immunity, then information about the true cumulative incidence can give insight into the extent of "herd immunity", and the effective reproductive number which depends on the fraction of the population that remains susceptible. Knowledge of cumulative incidence can help predict the timing of an epidemic peak under reopening scenarios and the extent of social distancing required to prevent a major surge of new infections in the future.

In order to design a valid seroprevalence study, researchers need to calculate the minimum sample size required to obtain an estimate that is sufficiently precise, requiring an estimate of the proportion of individuals who would test positive for antibodies. Model projections offer the opportunity to evaluate this quantity on future dates during which a proposed seroprevalence study might be conducted. Figure 4 shows projections of cumulative incidence among living individuals who might be sampled and tested in a future seroprevalence survey.

\section{Projections beyond Summer 2020}

In this report, we have focused on projecting incidence, hospitalizations, and deaths for Summer of 2020. Several factors make long-term projections challenging and potentially unreliable given currently available information about 

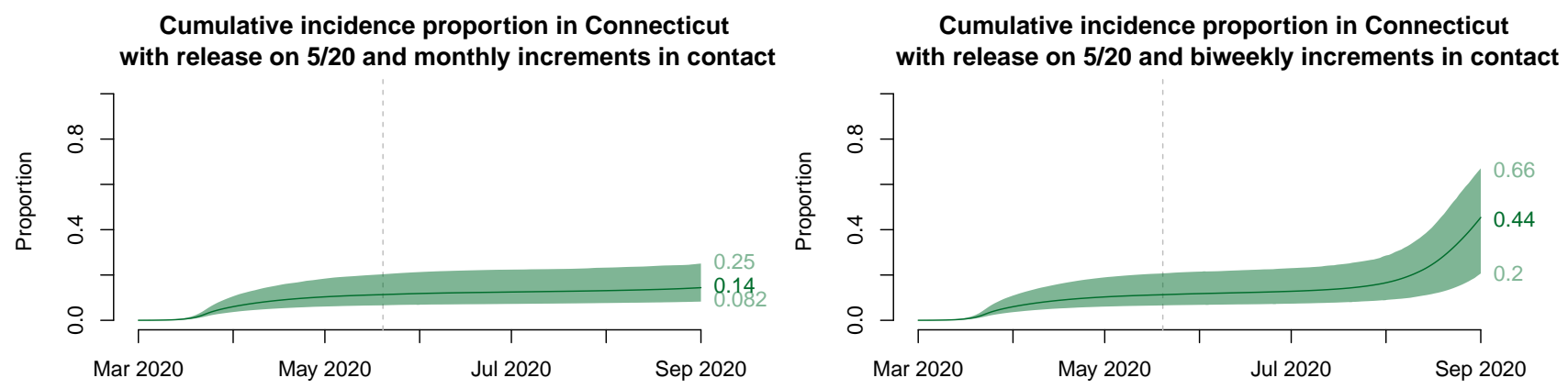

Figure 4: Cumulative incidence proportion among currently alive people under the "slow" (left) and "fast" (right) reopening scenario. Prospective planning of seroprevalence studies requires estimates like these of cumulative incidence estimated at future dates on which sampling might occur. The figure is modified from [28], doi: https: //doi.org/10.1101/2020.06.12.20126391.

the state's reopening plans, interventions, and the epidemiology of COVID-19. First, there is uncertainty in how contact patterns may change as a result of state and local policies. As nearby states reopen, and interstate travel increases, outbreaks outside of Connecticut may have a significant impact on incidence within Connecticut. It remains unclear whether schools and universities will reopen according to a standard or altered schedule in the Fall $[42,43]$. Second, epidemiologists do not yet know how infectious school-age children may be when they are infected, but it is known that children are unlikely to experience severe symptoms when infected with COVID-19 [23, 44-47]. Epidemiological studies have provided conflicting evidence regarding susceptibility, viral shedding and transmissibility in children [42, 48-50]. Because Connecticut closed K-12 schools approximately only one week before the stay-at-home order took effect, we do not have specific information about the isolated effect of closing schools on the subsequent reduction in transmission. If child care and summer camps reopen as planned at the end of June [51], we may be able to gain information about transmissibility in children from any subsequent increase in cases observed among people who are more likely to be symptomatic. This information will be useful in projecting COVID-19 dynamics in the Fall if K-12 education will take place in person. Third, we do not yet know whether COVID-19 transmission is sensitive to temperature or seasonal changes [34, 52]. If transmission is suppressed over the summer due to warmer temperatures, then Connecticut may enjoy low case counts and hospitalizations over the Summer, with a possible resurgence in the Fall as children return to school and temperatures cool. Another source of long-term uncertainty is related to potentially unequal depletion of susceptible individuals, depending on their risk profile. If a high proportion of older or otherwise high-risk people have already been infected, then we might expect a lower proportion of severe infections in the future, even if overall transmission remains high [53, 54]. Finally, the possibility of improved testing capacity, antibody tests, vaccines, or other therapeutics makes the effect of possible future public health and medical interventions difficult to predict.

Funding: We acknowledge NIH/NICHD grant 1DP2HD091799-01.

\section{Competing interests: None.}

Acknowledgements: We are grateful to Ted Cohen, Robert Heimer, and Edward H. Kaplan, for helpful comments. We thank the Connecticut Hospital Association for providing data on COVID-19 hospitalizations and deaths.

\section{References}

[1] Connecticut coronavirus map and case count. The New York Times. URL https://www.nytimes.com/interactive/ 2020/us/connecticut-coronavirus-cases.html.

[2] Totals by state. URL https://covidtracking.com/data\#state-ct. 
medRxiv preprint doi: https://doi.org/10.1101/2020.06.16.20126425; this version posted June 19, 2020. The copyright holder for this preprint (which was not certified by peer review) is the author/funder, who has granted medRxiv a license to display the preprint in perpetuity.

It is made available under a CC-BY 4.0 International license .

[3] Ned Lamont. Executive Order No. 7C: Protection of public health and safety during COVID-19 pandemic and response - further suspension or modification of statutes. State of Connecticut, March 15, 2020. URL https://portal.ct.gov/-/media/Office-of-the-Governor/Executive-Orders/Lamont-Executive-Orders/ Executive-Order-No-7C.pdf.

[4] Ned Lamont. Executive Order No. 7L: protection of public health and safety during COVID-19 pandemic and response - extension of school cancellation, municipal retiree reemployment, open fishing season and additional public health measures. March 24, 2020. URL https://portal.ct.gov/-/media/Office-of-the-Governor/ExecutiveOrders/Lamont-Executive-Orders/Executive-Order-No-7L.pdf.

[5] Ned Lamont. Executive Order No. 7X: Protection of public health and safety during COVID-19 pandemic and response - renter protections, extended class cancellation and other safety measures, educator certification, food trucks for truckers. April 10, 2020. URL https://portal.ct.gov/-/media/Office-of-the-Governor/ExecutiveOrders/Lamont-Executive-Orders/Executive-Order-No-7X.pdf.

[6] Ned Lamont. Executive Order No 7II: protection of public health and safety during COVID-19 pandemic and response - extension of school cancellation, home health care coverage, and food assistance measures. May 5, 2020. URL https://portal.ct.gov/-/media/Office-of-the-Governor/Executive-Orders/Lamont-ExecutiveOrders/Executive-Order-No-7II.pdf.

[7] Ned Lamont. Executive Order No. 7H: Protection of public health and safety during COVID-19 pandemic and response - restrictions on workplaces for non-essential businesses, coordinated response effort. March 20, 2020. URL https://portal.ct.gov/-/media/Office-of-the-Governor/Executive-Orders/Lamont-Executive-Orders/ Executive-Order-No-7H.pdf.

[8] Google. COVID-19 community mobility report: Connecticut. URL https://www.gstatic.com/covid19/mobility/ 2020-05-09_US_Connecticut_Mobility_Report_en.pdf.

[9] Facebook. Covid-19 mobility data network: Connecticut. URL https://visualization.covid19mobility.org/ ?date $=2020-05-15 \&$ dates $=2020-03-01 \_2020-05-15 \&$ region $=09$.

[10] Connecticut Department of Economic and Community Development. Sector rules and certification for May 20th reopen. URL https://portal.ct.gov/DECD/Content/Coronavirus-Business-Recovery/Sector-Rules-for-May-20Reopen. Accessed May 20, 2020.

[11] State of Connecticut. ContaCT: Connecticut contact tracing platform. Accessed May 5, 2020. URL https: //portal.ct.gov/Coronavirus/ContaCT.

[12] Connecticut Department of Public Health. COVID-19 update May 19, 2020. URL https://portal.ct.gov//media/Coronavirus/CTDPHCOVID19summary5192020.pdf.

[13] Ned Lamont. Governor Lamont announces suspension of regulations to support a major expansion of COVID19 testing. State of Connecticut, May 7, 2020. URL https://portal.ct.gov/Office-of-the-Governor/News/PressReleases/2020/05-2020/Governor-Lamont-Announces-Suspension-of-Regulations-to-Support-a-MajorExpansion-of-COVID19-Testing.

[14] Keith M. Phaneuf, Gregory B. Hladky, and Jenna Carlesso. Lamont calls for more testing as state gets closer to reopening some businesses. CT Mirror. URL https://ctmirror.org/2020/05/07/self-employed-residentsaffected-by-pandemic-could-receive-jobless-benefits-in-about-a-week/.

[15] Jacqueline Rabe Thomas. No surge yet for COVID-19 testing. Governor says it's coming. The CT Mirror, 2020. URL https://ctmirror.org/2020/05/05/no-surge-yet-for-covid-19-testing-governor-says-its-coming/.

[16] Stephen A Lauer, Kyra H Grantz, Qifang Bi, Forrest K Jones, Qulu Zheng, Hannah R Meredith, Andrew S Azman, Nicholas G Reich, and Justin Lessler. The incubation period of coronavirus disease 2019 (COVID-19) from publicly reported confirmed cases: estimation and application. Annals of Internal Medicine, 2020.

[17] Qifang Bi, Yongsheng Wu, Shujiang Mei, Chenfei Ye, Xuan Zou, Zhen Zhang, Xiaojian Liu, Lan Wei, Shaun A Truelove, Tong Zhang, Wei Gao, Cong Cheng, Xiujuan Tang, Xiaoliang Wu, Yu Wu, Binbin Sun, Suli Huang, Yu Sun, Juncen Zhang, Ting Ma, Justin Lessler, and Tiejian Feng. Epidemiology and transmission of COVID19 in 391 cases and 1286 of their close contacts in Shenzhen, China: a retrospective cohort study. The Lancet Infectious Diseases, 2020.

[18] Qun Li, Xuhua Guan, Peng Wu, Xiaoye Wang, Lei Zhou, Yeqing Tong, Ruiqi Ren, Kathy S.M. Leung, Eric H.Y. Lau, Jessica Y. Wong, Xuesen Xing, Nijuan Xiang, Yang Wu, Chao Li, Qi Chen, Dan Li, Tian 
medRxiv preprint doi: https://doi.org/10.1101/2020.06.16.20126425; this version posted June 19, 2020. The copyright holder for this preprint (which was not certified by peer review) is the author/funder, who has granted medRxiv a license to display the preprint in perpetuity.

It is made available under a CC-BY 4.0 International license .

Liu, Jing Zhao, Man Liu, Wenxiao Tu, Chuding Chen, Lianmei Jin, Rui Yang, Qi Wang, Suhua Zhou, Rui Wang, Hui Liu, Yinbo Luo, Yuan Liu, Ge Shao, Huan Li, Zhongfa Tao, Yang Yang, Zhiqiang Deng, Boxi Liu, Zhitao Ma, Yanping Zhang, Guoqing Shi, Tommy T.Y. Lam, Joseph T. Wu, George F. Gao, Benjamin J. Cowling, Bo Yang, Gabriel M. Leung, and Zijian Feng. Early transmission dynamics in Wuhan, China, of novel coronavirus-infected pneumonia. New England Journal of Medicine, 382(13):1199-1207, 2020.

[19] Natalie M Linton, Tetsuro Kobayashi, Yichi Yang, Katsuma Hayashi, Andrei R Akhmetzhanov, Sung-mok Jung, Baoyin Yuan, Ryo Kinoshita, and Hiroshi Nishiura. Incubation period and other epidemiological characteristics of 2019 novel coronavirus infections with right truncation: a statistical analysis of publicly available case data. Journal of Clinical Medicine, 9(2):538, 2020.

[20] Wenqing He, Y Yi Grace, and Yayuan Zhu. Estimation of the basic reproduction number, average incubation time, asymptomatic infection rate, and case fatality rate for COVID-19: Meta-analysis and sensitivity analysis. medRxiv, 2020.

[21] Henrik Salje, Cecile Tran Kiem, Noemie Lefrancq, Noemie Courtejoie, Paolo Bosetti, Juliette Paireau, Alessio Andronico, Nathanael Hoze, Jehanne Richet, Claire-Lise Dubost, Yann Le Strat, Justin Lessler, Daniel Levy Bruhl, Arnaud Fontanet, Lulla Opatowski, Pierre-Yves Boelle, and Simon Cauchemez. Estimating the burden of SARS-CoV-2 in France. medRxiv, 2020.

[22] Wycliffe E Wei, Zongbin Li, Calvin J Chiew, Sarah E Yong, Matthias P Toh, and Vernon J Lee. Presymptomatic transmission of SARS-CoV-2 - Singapore, January 23-March 16, 2020. Morbidity and Mortality Weekly Report, 69(14):411, 2020.

[23] Robert Verity, Lucy C Okell, Ilaria Dorigatti, Peter Winskill, Charles Whittaker, Natsuko Imai, Gina CuomoDannenburg, Hayley Thompson, Patrick GT Walker, Han Fu, Amy Dighe, Jamie T Griffin, Marc Baguelin, Sangeeta Bhatia, Anne Boonyasiri, Adhiratha andd Cori, Zulma Cucunubá, Rich FitzJohn, Katy Gaythorpe, Will Green, Arran Hamlet, Wes Hinsley, Daniel Laydon, Gemma Nedjati-Gilani, Steven Riley, Sabine van Elsland, Erik Volz, Haowei Wang, Yuanrong Wang, Xiaoyue Xi, Christl A Donnelly, Azra C Ghani, and Neil M Ferguson. Estimates of the severity of coronavirus disease 2019: a model-based analysis. The Lancet Infectious Diseases, 2020.

[24] Shikha Garg, Lindsay Kim, Michael Whitaker, Alissa O’Halloran, Charisse Cummings, Rachel Holstein, Mila Prill, Shua J Chai, Pam D Kirley, Nisha B Alden, Breanna Kawasaki, Kimberly Yousey-Hindes, Linda Niccolai, Evan J Anderson, Kyle P Openo, Andrew Weigel, Maya L Monroe, Patricia Ryan, Justin Henderson, Sue Kim, Kathy Como-Sabetti, Ruth Lynfield, Daniel Sosin, Salina Torres, Alison Muse, Nancy M Bennett, Laurie Billing, Melissa Sutton, Nicole West, William Schaffner, H. Keipp Talbot, Aquino Clarissa, Andrea George, Alicia Budd, Lynnette Brammer, Gayle Langley, Aron J Hall, and Alicia Fry. Hospitalization rates and characteristics of patients hospitalized with laboratory-confirmed coronavirus disease 2019 - COVID-NET, 14 states, March 1-30, 2020. MMWR. Morbidity and Mortality Weekly Report, 69, 2020.

[25] Eleanor M Rees, Emily S Nightingale, Yalda Jafari, Naomi Waterlow, Samuel Clifford, Carl A B Pearson, CMMID Working Group, Thibaut Jombert, Simon R Procter, and Gwenan M Knight. COVID-19 length of hospital stay: a systematic review and data synthesis. medRxiv, 2020.

[26] Joseph A Lewnard, Vincent X Liu, Michael L Jackson, Mark A Schmidt, Britta L Jewell, Jean P Flores, Chris Jentz, Graham R Northrup, Ayesha Mahmud, Arthur L Reingold, Maya Petersen, Nicholas P Jewell, Scott Young, and Jim Bellows. Incidence, clinical outcomes, and transmission dynamics of hospitalized 2019 coronavirus disease among 9,596,321 individuals residing in California and Washington, United States: a prospective cohort study. medRxiv, 2020.

[27] Ishan Paranjpe, Adam Russak, Jessica K De Freitas, Anuradha Lala, Riccardo Miotto, Akhil Vaid, Kipp W Johnson, Matteo Danieletto, Eddye Golden, Dara Meyer, Manbir Singh, Sulaiman Somani, Sayan Manna, Udit Nangia, Arjun Kapoor, Ross O’Hagan, Paul F O’Reilly, Laura M Huckins, Patricia Glowe, Arash Kia, Prem Timsina, Robert M Freeman, Matthew A Levin, Jeffrey Jhang, Adolfo Firpo, Patricia Kovatch, Joseph Finkelstein, Judith A Aberg, Emilia Bagiella, Carol R Horowitz, Barbara Murphy, Zahi A Fayad, Jagat Narula, Eric J Nestler, Valentin Fuster, Carlos Cordon-Cardo, Dennis S Charney, David L Reich, Allan C Just, Erwin P Bottinger, Alexander W Charney, Benjamin S Glicksberg, Girish Nadkarni, and on behalf of the Mount Sinai Covid Informatics Center (MSCIC). Clinical characteristics of hospitalized COVID-19 patients in New York 
medRxiv preprint doi: https://doi.org/10.1101/2020.06.16.20126425; this version posted June 19, 2020. The copyright holder for this preprint (which was not certified by peer review) is the author/funder, who has granted medRxiv a license to display the preprint in perpetuity.

It is made available under a CC-BY 4.0 International license .

City. medRxiv, 2020.

[28] Olga Morozova, Zehang Richard Li, and Forrest W. Crawford. A model for COVID-19 transmission in Connecticut. medRxiv, 2020. URL https://github.com/fcrawford/covid19_model_report/releases.

[29] Connecticut State Department of Public Health. COVID-19 Daily DPH Reports Library, 2020. URL https: //data.ct.gov/Health-and-Human-Services/COVID-19-Daily-DPH-Reports-Library/bqve-e8um.

[30] Connecticut Hospital Association, 2020. URL https://cthosp.org/.

[31] CHIMEData, 2020. URL https://chimedata.org/.

[32] State of Connecticut. Coronavirus disease 2019 (covid-19). URL https://portal.ct.gov/Coronavirus.

[33] Ruiyun Li, Sen Pei, Bin Chen, Yimeng Song, Tao Zhang, Wan Yang, and Jeffrey Shaman. Substantial undocumented infection facilitates the rapid dissemination of novel coronavirus (SARS-CoV2). Science, 2020.

[34] Stephen M Kissler, Christine Tedijanto, Edward Goldstein, Yonatan H Grad, and Marc Lipsitch. Projecting the transmission dynamics of SARS-CoV-2 through the postpandemic period. Science, 2020.

[35] Roman Wölfel, Victor M. Corman, Wolfgang Guggemos, Michael Seilmaier, Sabine Zange, Marcel A. Müller, Daniela Niemeyer, Terry C. Jones, Patrick Vollmar, Camilla Rothe, Michael Hoelscher, Tobias Bleicker, Sebastian Brünink, Julia Schneider, Rosina Ehmann, Katrin Zwirglmaier, Christian Drosten, and Clemens Wendtner. Virological assessment of hospitalized patients with COVID-2019. Nature, pages 1-5, 2020.

[36] Hiroshi Nishiura, Tetsuro Kobayashi, Takeshi Miyama, Ayako Suzuki, Sung-Mok Jung, Katsuma Hayashi, Ryo Kinoshita, Yichi Yang, Baoyin Yuan, Andrei R Akhmetzhanov, and Natalie M Linton. Estimation of the asymptomatic ratio of novel coronavirus infections (COVID-19). International Journal of Infectious Diseases, 94:154 - 155, 2020.

[37] Kenji Mizumoto, Katsushi Kagaya, Alexander Zarebski, and Gerardo Chowell. Estimating the asymptomatic proportion of coronavirus disease 2019 (COVID-19) cases on board the Diamond Princess cruise ship, Yokohama, Japan, 2020. Eurosurveillance, 25(10):2000180, 2020.

[38] Jon C Emery, Timothy W Russel, Yang Liu, Joel Hellewell, Carl AB Pearson, CMMID 2019-nCoV working group, Gwen M Knight, Rosalind M Eggo, Adam J Kucharski, Sebastian Funk, Stefan Flasche, and Rein M G J Houben. The contribution of asymptomatic SARS-CoV-2 infections to transmission-a model-based analysis of the Diamond Princess outbreak. medRxiv, 2020.

[39] Enrico Lavezzo, Elisa Franchin, Constanze Ciavarella, Gina Cuomo-Dannenburg, Luisa Barzon, Claudia Del Vecchio, Lucia Rossi, Riccardo Manganelli, Arianna Loregian, Nicolò Navarin, Davide Abate, Manuela Sciro, Stefano Merigliano, Ettore Decanale, Maria Cristina Vanuzzo, Francesca Saluzzo, Francesco Onelia, Monia Pacenti, Saverio Parisi, Giovanni Carretta, Daniele Donato, Luciano Flor, Silvia Cocchio, Giulia Masi, Alessandro Sperduti, Lorenzo Cattarino, Renato Salvador, Katy A.M. Gaythorpe, Imperial College London COVID-19 Response Team, Alessandra R Brazzale, Stefano Toppo, Marta Trevisan, Vincenzo Baldo, Christl A. Donnelly, Neil M. Ferguson, Ilaria Dorigatti, and Andrea Crisanti. Suppression of COVID-19 outbreak in the municipality of Vo, Italy. medRxiv, 2020.

[40] Katherine H. Campbell, Jean M. Tornatore, Kirsten E. Lawrence, Jessica L. Illuzzi, L. Scott Sussman, Heather S. Lipkind, and Christian M. Pettker. Prevalence of SARS-CoV-2 among patients admitted for childbirth in Southern Connecticut. JAMA, 052020.

[41] Elizabeth A Lane, Damien J Barrett, Miriam Casey, Conor G McAloon, Aine B Collins, Kevin Hunt, Andrew W Byrne, David McEvoy, Ann Barber, John M Griffin, Patrick Wall, and Simon J More. Country differences in hospitalisation, length of stay and admission to Intensive Care Units due to SARS-CoV-2 infection: a rapid review of available literature. medRxiv, 2020.

[42] Russell M Viner, Simon J Russell, Helen Croker, Jessica Packer, Joseph Ward, Claire Stansfield, Oliver Mytton, Chris Bonell, and Robert Booy. School closure and management practices during coronavirus outbreaks including COVID-19: a rapid systematic review. The Lancet Child \& Adolescent Health, 2020.

[43] Daniel Mangrum and Paul Niekamp. College student contribution to local COVID-19 spread: Evidence from university spring break timing (May 20, 2020). SSRN, 2020.

[44] Jonas F Ludvigsson. Systematic review of COVID-19 in children shows milder cases and a better prognosis than adults. Acta Paediatrica, 109(6):1088-1095, 2020.

[45] Daniel F. Gudbjartsson, Agnar Helgason, Hakon Jonsson, Olafur T. Magnusson, Pall Melsted, Gudmundur L. 
medRxiv preprint doi: https://doi.org/10.1101/2020.06.16.20126425; this version posted June 19, 2020. The copyright holder for this preprint (which was not certified by peer review) is the author/funder, who has granted medRxiv a license to display the preprint in perpetuity.

It is made available under a CC-BY 4.0 International license .

Norddahl, Jona Saemundsdottir, Asgeir Sigurdsson, Patrick Sulem, Arna B. Agustsdottir, Berglind Eiriksdottir, Run Fridriksdottir, Elisabet E. Gardarsdottir, Gudmundur Georgsson, Olafia S. Gretarsdottir, Kjartan R. Gudmundsson, Thora R. Gunnarsdottir, Arnaldur Gylfason, Hilma Holm, Brynjar O. Jensson, Aslaug Jonasdottir, Frosti Jonsson, Kamilla S. Josefsdottir, Thordur Kristjansson, Droplaug N. Magnusdottir, Louise le Roux, Gudrun Sigmundsdottir, Gardar Sveinbjornsson, Kristin E. Sveinsdottir, Maney Sveinsdottir, Emil A. Thorarensen, Bjarni Thorbjornsson, Arthur Löve, Gisli Masson, Ingileif Jonsdottir, Alma D. Möller, Thorolfur Gudnason, Karl G. Kristinsson, Unnur Thorsteinsdottir, and Kari Stefansson. Spread of SARS-CoV-2 in the Icelandic population. New England Journal of Medicine, 2020.

[46] Yuanyuan Dong, Xi Mo, Yabin Hu, Xin Qi, Fan Jiang, Zhongyi Jiang, and Shilu Tong. Epidemiology of COVID-19 among children in China. Pediatrics, 2020.

[47] Adam S Dingens, Katharine HD Crawford, Amanda Adler, Sarah L Steele, Kirsten Lacombe, Rachel Eguia, Fatima Amanat, Alexandra C Walls, Caitlin R Wolf, Michael Murphy, Deleah Pettie, Lauren Carter, Xuan Qin, Neil P King, David Veesler, Florian Krammer, Helen Y Chu, Janet A Englund, and Jesse D Bloom. Seroprevalence of SARS-CoV-2 among children visiting a hospital during the initial Seattle outbreak. medRxiv, 2020.

[48] Ping-Ing Lee, Ya-Li Hu, Po-Yen Chen, Yhu-Chering Huang, and Po-Ren Hsueh. Are children less susceptible to COVID-19? Journal of Microbiology, Immunology, and Infection, 2020.

[49] T Jones, Barbara Mühlemann, Talitha Veith, Marta Zuchowski, Jörg Hofmann, Angela Stein, Anke Edelmann, V Corman, and Christian Drosten. An analysis of SARS-CoV-2 viral load by patient age. German Research network Zoonotic Infectious Diseases website, 2020.

[50] Laura Di Domenico, Giulia Pullano, Chiara E Sabbatini, Pierre-Yves Boëlle, and Vittoria Colizza. Expected impact of reopening schools after lockdown on COVID-19 epidemic in Île-de-France. medRxiv, 2020.

[51] Susan Dunne. State officials are allowing Connecticut summer camps to open June 29, but some still aren't sure if they will. Hartford Courant, May 6, 2020. URL https://www.courant.com/coronavirus/hc-news-coronavirussummer-camps-opening-20200506-hgiigsmiljfdtfnre37kgbk7yi-story.html.

[52] Ran Xu, Hazhir Rahmandad, Marichi Gupta, Catherine DiGennaro, Navid Ghaffarzadegan, Heresh Amini, and Mohammad S Jalali. The modest impact of weather and air pollution on COVID-19 transmission. medRxiv, 2020.

[53] M Gabriela M Gomes, Ricardo Aguas, Rodrigo M Corder, Jessica G King, Kate E Langwig, Caetano SoutoMaior, Jorge Carneiro, Marcelo U Ferreira, and Carlos Penha-Goncalves. Individual variation in susceptibility or exposure to SARS-CoV-2 lowers the herd immunity threshold. medRxiv, 2020.

[54] Tom Britton, Frank Ball, and Pieter Trapman. The disease-induced herd immunity level for Covid-19 is substantially lower than the classical herd immunity level. arXiv preprint arXiv:2005.03085, 2020. 